Aims. Staff mental health is a major determinant of a wellfunctioning health system that has become ever more important during the COVID-19 pandemic. Poor mental health is the most common reason for NHS staff sickness absences, usually accounting for $25 \%$ of all reported sick leave. At a time when the NHS most needs an available and efficient workforce, government and NHS employers lack the necessary evidence to inform decisions about how best to support the mental health needs of its staff. In this report, we share our experience and the initial figures from a newly developed multidisciplinary assessment and treatment service for NHS staff.

Method. The Staff Mental Health Service (SMHS) at the Cambridgeshire and Peterborough NHS Foundation Trust (CPFT) launched in September 2020. The SMHS is commissioned by the Cambridgeshire and Peterborough sustainability and transformation partnership and is accessible to the 25,513 staff based at five NHS trusts within the region. The service received 235 referrals within 5 months of the launch. All patients had a first clinical contact within three working days and more than $80 \%$ had their initial assessment within two weeks. The SMHS clinical team is comprised of consultant psychiatrists, senior clinical psychologists, specialist mental health nurses, and an occupational health nurse set to provide rapid access, confidential, evidence-based treatments for the NHS staff. As part of service evaluation within CPFT, we collected routine screening data (Patient Health Questionnaire-9 (PHQ-9), Generalised Anxiety Disorder-7 (GAD-7), and Posttraumatic Symptom Check List - 6 (PCL-6)) from patients completing the initial assessment $(n=130)$.

Result. According to the initial figures $(n=130)$ from a diverse group of healthcare staff, on average the patients presented with moderate level of depressive symptoms (PHQ-9: $16.22 \pm 5.94$ ). Anxiety levels were in moderate to severe range (GAD-7: 13.45 $\pm 4.70)$. Average score of PCL-6 checklist for traumatic stress symptoms was higher than the established cut-off $(>14): 19.43$ \pm 5.65 .

Conclusion. The Staff Mental Health Service offers an innovative, multi-disciplinary rapid assessment and treatment clinic for NHS staff. The demand for the service has been immense, reaching double the number of predicted referrals. Initial data suggested high rates of moderate to severe depression, anxiety, and traumatic stress symptoms in healthcare workers. Our clinical observations was that many healthcare workers have had longstanding significant mental health conditions that saw deterioration during the COVID-19 pandemic. We hope that our experience in the SMHS will help inform models across the UK to address the clear unmet need for staff mental health.

\section{Profile of sleep pattern, psychiatric comorbidity and problematic electronic gadget use in children and adolescents with autism and ADHD}

Darpan Kaur ${ }^{1 \star}$, Sanay Patani ${ }^{2}$, Rishab Verma ${ }^{1}$ and Rakesh Ghildiyal ${ }^{1}$

${ }^{1}$ Department of Psychiatry, Mahatma Gandhi Missions Medical College and Hospital, Sector 01, Kamothe, Navi Mumbai, Maharashtra, India and ${ }^{2}$ Mahatma Gandhi Missions Medical College and Hospital, Sector 01, Kamothe, Navi Mumbai, Maharashtra, India ${ }^{*}$ Corresponding author.

doi: 10.1192/bjo.2021.143

Aims. To assess the profile of Sleep pattern, Psychiatric comorbidity and problematic electronic gadget use and explore demographic factors and correlations in children and adolescents with ADHD and Autism.
Hypothesis: There are statistically significant problems and associations across sleep pattern, psychiatric comorbidity and gadget use in children and adolescents with autism and ADHD. Background. Literature highlights increasing global trends and emerging concerns over the problematic use of electronic gadgets and sleep related problems in children and adolescents with autism and ADHD. There is sparse literature on the profile of sleep patterns, psychiatry comorbidity and problematic gadget use in children and adolescents with autism and ADHD from developing countries.

Method. This was an observational study conducted at the Child and Adolescent Psychiatry Clinic, Department of Psychiatry at a tertiary care Institution under the STS ICMR Project 2019 with Institutional Ethics Clearance. Apriori Sample size calculated was 70. Children and adolescents diagnosed with autism or ADHD as per ICD 10 criteria, fulfilling the inclusion criteria and willing to participate in the study were included. Informed consent was obtained from caregivers. Sleep Disturbance Scale for Children, Self Designed Parent based Problematic Electronic Gadget Use Scale, Vanderbilt ADHD scale, Indian scale for Assessment of Autism and the Child and Adolescent Psychiatry Clinic structured Performa were the tools for data collection. The results were analyzed with descriptive tests, chi square test and multiple logistic regressions using SPSS.

Result. Mean age of the sample was 9.1 years and majority (57\%) were boys. Forty nine patients had ADHD and 21 patients had Autism. Problematic gadget use was higher in children ranging from 6 to 15 years of age and $12.8 \%$ had severe levels of problematic gadget use. $34.3 \%$ patients experienced severe problems in initiating and maintaining sleep. Oppositional-Defiant disorder was the most common comorbidity, predominantly inattentive type (76.4\%) was the most common subtype of ADHD and mild autism $(54.3 \%)$ was the most common type of autism in the sample. There were statistically significant associations $(p<0.05)$ between age and gadget use; hyperactive subtype of ADHD and problems with initiating and maintaining sleep and ADHD subtype, sex profile and problematic gadget use.

Conclusion. We conclude that sleep problems, psychiatric comorbidity and problematic gadget use are prevalent with statistically significant associations in children and adolescents with autism and ADHD as per our study findings. Our study has relevant clinical, research and policy implications.

Pregnancy audit in the PATH (Psychosis Prevention, Assessment and Treatment in Hertfordshire) early intervention in psychosis service

Jessica Kershaw*, Zohra Taousi, Hildah Jiah, Giovanni Borghini, Debbi Knight and Keith Brown

Hertfordshire Partnership University NHS Foundation Trust ${ }^{\star}$ Corresponding author.

doi: 10.1192/bjo.2021.144

Aims. To identify whether staff from the PATH Early Intervention in Psychosis (EIP) Service routinely ask female service users of child bearing age about their plans for pregnancy, whether risks of medication in pregnancy are routinely discussed and whether contraception is routinely discussed.

Method. In May 2019, a report was run capturing all female PATH service users of child bearing age (16-50 years) who were on the pathway at this time. This totalled 177 service users, all of whom were included in the sample. We used the search terms "pregnant", "pregnancy", "having children", "contraception", “conceive", "baby", "conception”, "miscarriage", "abortion”, 